\title{
MONITORING OF SURFACE SUBSIDENCE OF THE MINING AREA BASED ON SBAS
}

\author{
Yufeng Zhu, Shijian Zhou, Deyan Zang, Tieding Lu \\ Faculty of Geomatics, East China Institute of Technology, NanChang, China - zyf_215@163.com
}

Commission III, WG III/3

KEY WORDS: D-InSAR, Small-baseline Subset, Mining subsidence, deformation monitoring

\begin{abstract}
:
This paper has collected 7 scenes of L band PALSAR sensor radar data of a mine in FengCheng city, jiangxi province, using the Small-baseline Subset (SBAS) method to invert the surface subsidence of the mine. Baselines of interference less than $800 \mathrm{~m}$ has been chosen to constitute short baseline differential interference atlas, using pixels whose average coherent coefficient was larger than or equal to 0.3 as like high coherent point target, using singular value decomposition (SVD) method to calculate deformation phase sequence based on these high coherent points, and the accumulation of settlements of study area of different period had been obtained, so as to reflect the ground surface settlement evolution of the settlement of the area. The results of the study has showed that: SBAS technology has overcome coherent problem of the traditionality D-InSAR technique, continuous deformation field of surface mining in time dimension of time could been obtained, characteristics of ground surface settlement of mining subsidence in different period has been displayed, so to improve the accuracy and reliability of the monitoring results.
\end{abstract}

\section{INTRODUCTION}

Ground settlement and subsidence caused by mining is a destruction disasters often happening in mining area, and it is one of the most important geological disasters in China. Ground subsidence of mined-out area not only destroys all kinds of architectural, engineering, water conservancy, transportation facilities and farmland, it is also a threat to people's life and property safety, and it has become an important factor which influences and restricts the sustainable development of mining cities (Yufeng Zhu et al., 2012) and (Yufeng Zhu et al., 2011). Therefore, it is necessary to use the advanced technology to monitor and control damage caused by the ground surface settlement, which can guarantee the sustainable development of mining area.

Differential Interferometric Synthetic Aperture echoes(DInSAR) is a surface deformation monitoring method recently developed, monitoring the coal mine area of all-weather, noncontact, planar, fast and accurate, with centimeters or mm level. However D-InSAR technology focuses on the single deformation within short time interval, so SAR image which can be used is little, without redundant observations. When the deformation phase is not far from phase noise, deformation phase can't be separated effectively, and reliability of deformation calculation is poor (Hongjie Yin et al., 2011). Because of incoherent of the time and the baselines, orbit error and the influence of the atmosphere, D-InSAR single discrete settlements cannot be connected in most cases, and it is difficult to detect the deformation sequence, and the development and evolution of settlement of the study area coal mine can't be obtained.

According to the D-InSAR technology limitations, in recently years, some scholars abroad calculated the deformation rate effectively by using least squares estimation method in the high coherence point target, through virtual observation method, and in which accuracy and reliability of D-InSAR technology deformation calculation has been improved(Usai et al., 1999) and (Usai et al., 2003) . Ferretti, etc. first proposed Permanent
Scatter technology, choosing a series of phase stable point as PS point according to a certain criterion in a group of radar interference image given, and after removing DEM error, atmospheric delay phase, orbit error and other noise of PS points according to the given phase model, then the exact deformation of surface shape can be obtained (Ferretti et al., 2000) and (Ferretti et al., 2001).

Berardino (Berardino et al., 2002) and Lanari (Lanari et al., 2004) proposed SBAS method, using small baselines combination to get interference measurement, calculating of the combination of much small baselines using SVD method can effectively to solve discontinuous time problems caused by space between baseline of different SAR data which is too long to improve resolution of monitoring time, so that accuracy of DInSAR technology deformation calculation has been improved further.

In this paper, a coal mine area in FengCheng city, Jiangxi province has been selected as the research area, the L-band PALSAR data of the region has been chosen to do time series analysis using SBAS technology, and time series deformation field could be obtained, and mining subsidence funnel could be detected and positioned. Development situation of mining subsidence funnel has been analyzed, to reveal the rule of evolution of subsidence funnel according with time (Ferretti et al., 2000) and (Ferretti et al., 2001) .

\section{EXPERIMENTAL RESEARCH}

In order to verify the validity and practicability of the technology in surface subsidence monitoring, a coal mine area in FengCheng city, Jiangxi province has been chosen as the research object, using SBAS technology for time series analysis, sedimentation sequence diagram from June 2007 to May 2008 could be obtained.

\subsection{The experimental datas}

In this paper, 7 scenes the L-band SAR datas of PALSAR 
ALOS satellite sensors covered plateau lake coal mine area and a series of disposal of no "empty" global SRTM V4 DEM data with 3 seconds accuracy (resolution for $90 \mathrm{~m}$ ) which nominal absolute elevation accuracy is $\pm 16 \mathrm{~m}$ and nominal absolute plane precision is $\pm 20 \mathrm{~m}$ provided by spatial information association (CSI) of CGIAR were used. The specific parameters of image information are shown in table 1 . In the table image of FBD mode should be 2 times over sampling in the distance in order to keep consistent resolution with FBS mode. In removing topographic phase information with collecting DEM, it should do 6 times over sampling of DEM.

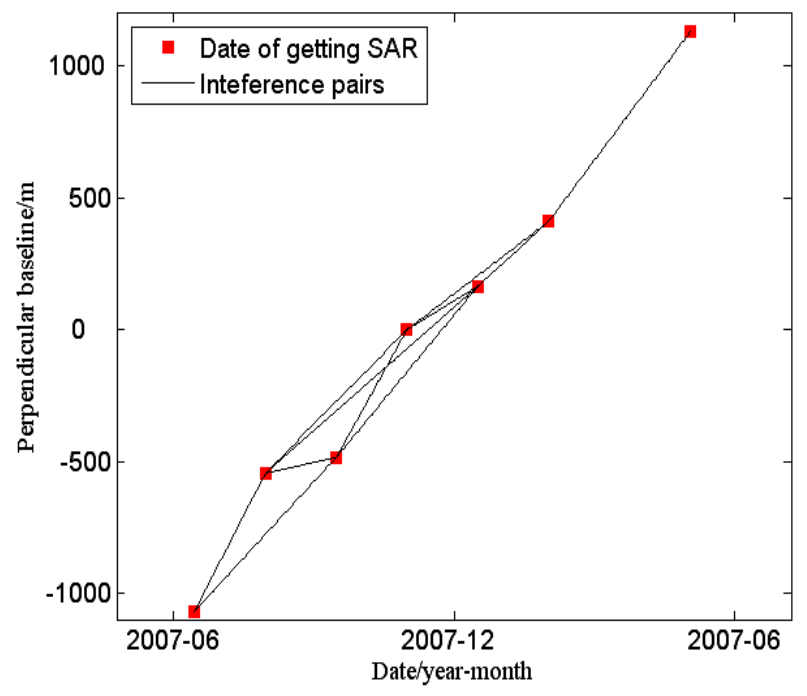

Figure 1. The schedule of SAR data

\subsection{Data processing}

Using SBAS method to process data, firstly SAR image data acquired were done with differential interference preproccess of D-InSAR method, and time series D-InSAR analysis model were established to calculate settlement rate and cumulative type variable of each time based on selected high coherent points.

2.2.1 Pretreatment: Before the D-InSAR time series analysis, small base set interferogram sequence was generated by SAR data "two rail" method differential interference processing in GAMMA software of Swiss GAMMA Company in this paper.

\section{(1) Selection of IE image}

Because SBAS method is used to analyze the time series of identical point, as PS technology, interferograms should be registered to the same coordinate system in this method. Therefore, a picture of IE image should be chosen, and other images should be registered to the IE image, and then single visual interference figure could be generated based on the same main image. The image of the vertical baseline less than $800 \mathrm{~m}$ of interference and the time base, vertical baseline and doppler centroid frequency baseline of minimizing was chosen as IE image. IE image selected in this paper was SAR image obtained in October 30, 2007.

\begin{tabular}{|c|c|c|c|c|}
\hline $\begin{array}{c}\text { Image } \\
\text { Number }\end{array}$ & $\begin{array}{c}\text { Sum } \\
\text { of } \\
\text { Time }\end{array}$ & $\begin{array}{c}\text { Sum of } \\
\text { Vertical } \\
\text { Baseline }\end{array}$ & $\begin{array}{c}\text { Sum of } \\
\text { Doppler } \\
\text { frequency }\end{array}$ & $\begin{array}{c}\text { Sum of } \\
\text { Three } \\
\text { Baselines }\end{array}$ \\
\hline 20070614 & 1012 & 7085.1524 & 45.3596 & 8142.5120 \\
\hline
\end{tabular}

\begin{tabular}{|c|c|c|c|c|}
\hline 20070730 & 782 & 4467.0160 & 44.5616 & 5293.5776 \\
20070914 & 644 & 4288.3927 & 74.4414 & 5006.8341 \\
20071030 & 598 & 3803.8635 & 51.6112 & 4453.4747 \\
20071225 & 644 & 3967.3606 & 86.5655 & 4697.9261 \\
20080130 & 782 & 4701.1704 & 66.9226 & 5550.0930 \\
20080501 & 1242 & 8304.1775 & 44.1424 & 9590.3199 \\
\hline
\end{tabular}

Table 1. The statistical table about three baselines

For the L-band of ALOS satellite, less than or equal to $800 \mathrm{~m}$ of the baseline can be used as a small baseline, the space of incoherent could be improved, and the coherence increased obviously (Hongjie Yin et al., 2011). Similarly, L band PALSAR data were selected in this experiment, baseline less than $800 \mathrm{~m}$ were selected to produce 11 small baselines interfere pairs, and with the use of SBAS method.

\section{(2) Generation of differential interference pattern}

Interferogram was generated by SLC differential interference in GAMMA commercial software Swiss GAMMA Company. This process was the foundation of D-InSAR time series analysis. Specific operation processment was as follows:

IE image should be cut, the scope should include the study area and have obvious relief change, and it is an advantage to the registration of study area of SAR intensity map simulated by DEM and IE image intensity map. In this paper the size of the cut was 7000 pixels in the distance, 17500 pixels in the bearing;

The other image was registered to IE image, and single visual interferogram was generated based on the same IE image;

The small base set interference was registered another, generating more apparent small baseline interference atlas of 5 bearing, 2 distance (image size: 3500 pixel $* 3500$ pixel); Then the small baseline interference atlas was interfered using two rail differential interference and filtering, and then differential interference atlas could be obtained after filter; Further utilizing the MCF method for unwrapping differential interference graph, then unwrapping differential interference atlas under radar coordinates could be obtained;

Due to orbit data of PALSAR radar image was not precise, similar "the ground effect" of the interference fringes (track error phase) was concluded in the filtered differential interference. Unwrapping differential interference figure of orbit error phase removed could be obtained by difference interferogram unwrapping minus orbit error phrase, using the least squares fitting method in this paper.

The least squares solution was:

$$
A=\left(M^{\prime} M\right)^{-1} M^{\prime} \phi
$$

Differential interference atlas unwrapping in the geographic coordinates could be obtained by geocoding of the differential interference graph unwrapping which has removed the orbit error.

Due to the small mining area, in order to reflect the study area of the surface settlement clearly and reduce the workload, data processing using only a small image map (550 pixel* $^{*} 400$ pixel) was inversion calculated later. 
The unwrapping phase atlas of small range geographic coordinates was shown in figure 2.

From the unwrapping phase figure 2, the subsidence area of mining area surface was clear seen, and there was unwrapping deviation in the dotted area from the interference of 20070914 20070614, and it was due to the bigger baseline of the interference. And in the same time the field was in summer, the surface vegetation was lush growth, which was leading to the poor coherence of interference. Also there was unwrapping deviation in the dotted area from the interference of 20080130 20071030, this interference was in winter, plant was rare, which would not be affected by time incoherent, but during 15 December 2007 to 2008 on January 30, the study area was appeared to "frozen" disaster, which affected the radar echo signal this period, causing that coherence of interference was very poor, interference phase was discontinuity, thus there were some errors in unwrapping results.

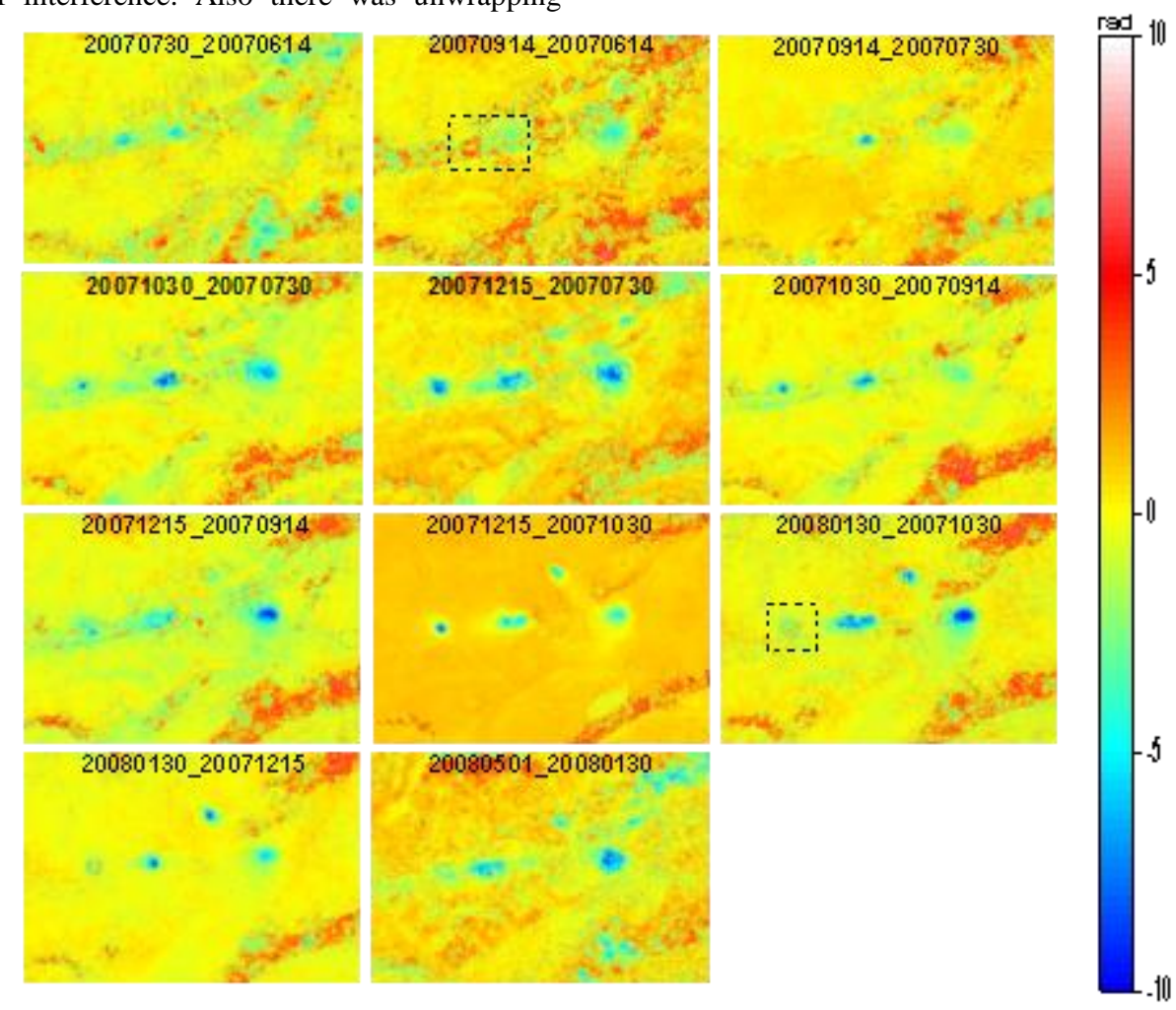

Figure 2.The unwrapped interferograms in MCF

2.2.2 Choice of high coherent targets: Before calculating deformation rate and DEM error by use of SBAS time series analysis model, the first was to choose the high coherent target. The chosen of the high coherent points would be able to make data of practical processing later reduced greatly, from a few bit down to hundreds of bits. At present the methods of selection of high coherent target are amplitude threshold value method and correlation coefficient threshold value method. From the experimental, density of high coherent point by the coherent coefficient threshold value method was bigger than density of high coherent point by the amplitude threshold value method (Xiaoyan Dong, 2011).Amplitude threshold value method is used to estimate phase stability using pixel strength stability instead of coherence, so this often needs more SAR image (generally not less than 30 picture), and the statistical characteristic of the amplitude can be estimated correct. And in time series, stable high coherent point could be found by analyzing the strength of the pixel, which involves complicated mathematics method, and need do a large number of simulation experiments to determine the threshold value. Due to the small amount of data in this paper was small, simple algorithm coherent coefficient threshold value method was chosen to selected high coherent point. Selection criteria: coherent map of time series was taken average, the threshold value was chosen by average coherent coefficient of the cumulative images. Namely:

$$
\gamma_{\text {mean }}=\frac{1}{M} \sum_{j-1}^{M} \gamma_{j} \geq \gamma^{T}
$$

2.2.3 SBAS processing: In the selection of high coherent target, a linear deformation rate of the linear model and constructs the system of equations is adopted in this paper, the method of singular value decomposition (SVD) to solution equations, it is estimated that interval linear settlement rate each time. Each time settlement rate in time domain integral can get on the accumulation of each time form variables.

\subsection{Results analysing}

Differential interference atlas unwrapping of study region after geocoding has been analyzed by SBAS method, and each time of settling diagram of mining area surface could be obtained, as shown in figure 3. 


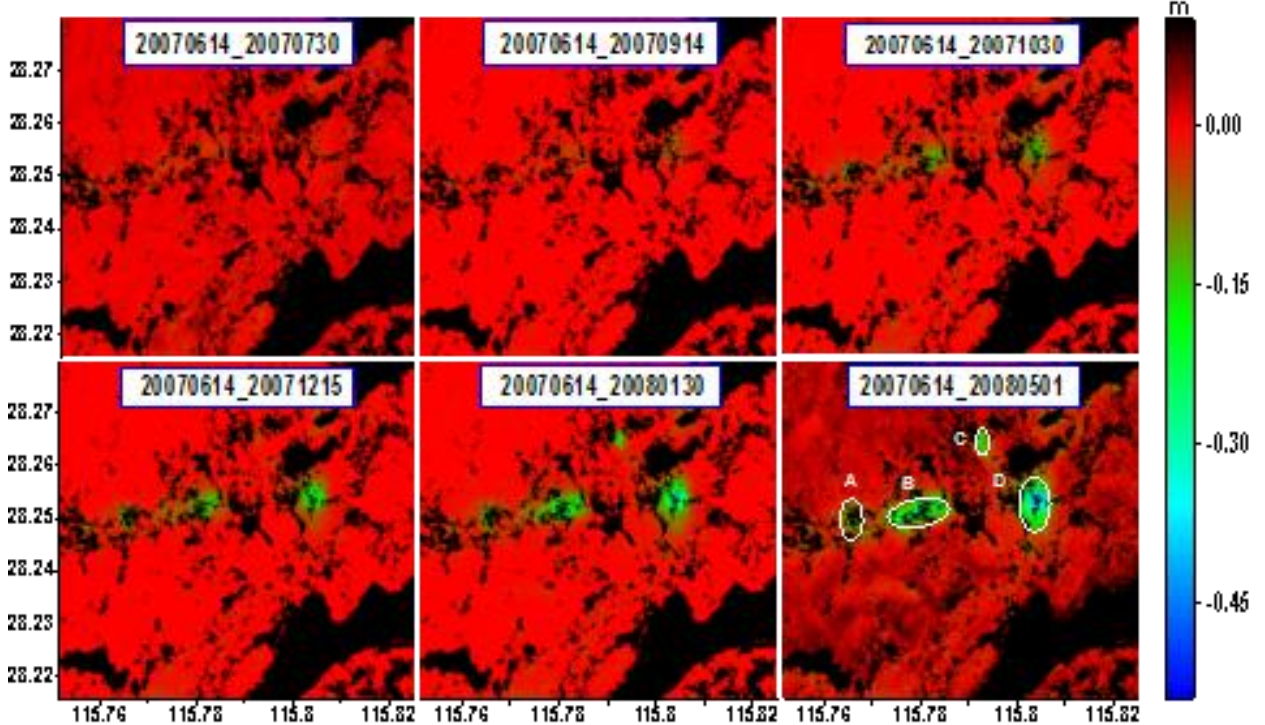

Figure 3.Time series settlement map of study region

From figure 3 it could be seen that the settlement and settlement area of mining area surface has been growing, forming subsidence funnel $\mathrm{A}, \mathrm{B}, \mathrm{C}$ and $\mathrm{D}$ during this period of time. The accumulated settlement and subsidence area of subsidence funnel $\mathrm{A}$ and $\mathrm{C}$ were small in this period of time, because of slow settlement in early production period; Accumulated settlement of subsidence funnel $\mathrm{B}$ and $\mathrm{C}$ were bigger in this period of time, because of sedimentation acceleration in mining active phase. The accumulation of subsidence area in different period should be statistical analyzed, and the results of statistical could be seen in table 2 . It could be clearly seen in table 2 that subsidence area of the study area was gradually expanding as times goes on. Due to the influence of ice disaster the SAR image of January 30, 2008, overall coherence of this group of image combination of the differential interferogram was very poor.

\begin{tabular}{|c|c|c|c|c|c|}
\hline \multirow{2}{*}{ Date } & \multicolumn{5}{|c|}{ Subsidence area $\left(\right.$ Unit: $\left.^{2}\right)$} \\
\cline { 2 - 6 } & $\begin{array}{l}\text { Settle } \\
\text { ment } \\
>0.1 \mathrm{~m}\end{array}$ & $\begin{array}{l}\text { Settle } \\
\text { ment } \\
>0.2 \mathrm{~m}\end{array}$ & $\begin{array}{l}\text { Settle } \\
\text { ment } \\
>0.3 \mathrm{~m}\end{array}$ & $\begin{array}{l}\text { Settle } \\
\text { ment } \\
>0.4 \mathrm{~m}\end{array}$ & $\begin{array}{l}\text { Settle } \\
\text { ment } \\
>0.5 \mathrm{~m}\end{array}$ \\
\hline 20070730 & 591.0588 & 0 & 0 & 0 & 0 \\
20070914 & 1418.5000 & 0 & 0 & 0 & 0 \\
20071030 & 104970 & 0 & 0 & 0 & 0 \\
20071215 & 219050 & 9693.4000 & 0 & 0 & 0 \\
20080130 & 408890 & 7565.6000 & 7683.8000 & 0 & 0 \\
20080501 & 762580 & 190680 & 59106 & 12058 & 470.8470 \\
\hline
\end{tabular}

Table 2. Accumulated subsidence area

In order to analyze the development of subsidence funnel further, subsidence funnel $\mathrm{A}, \mathrm{C}$ and $\mathrm{D}$ has been selected to do profile analysis, in order to observe their time series of the changes of the settlement.

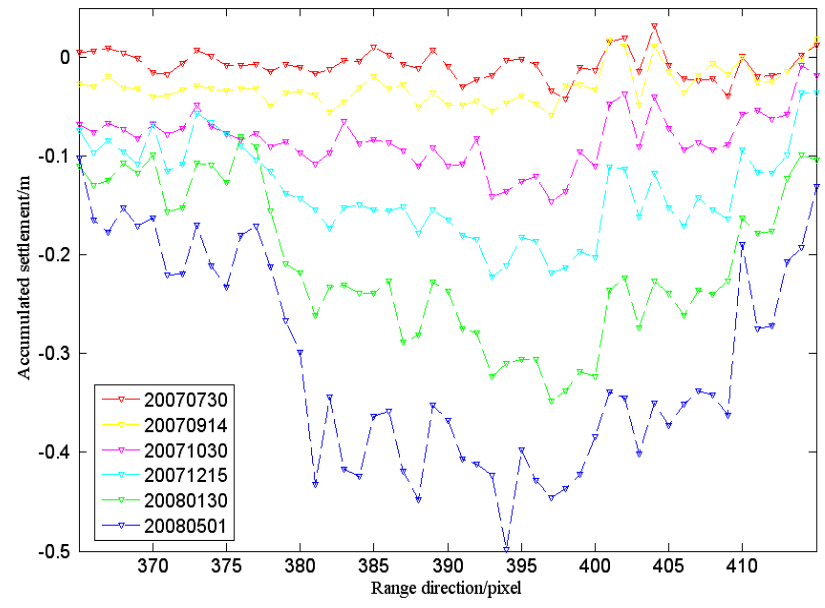

Figure 4.Time series settlement profiles of subsidence funnel D in Range direction

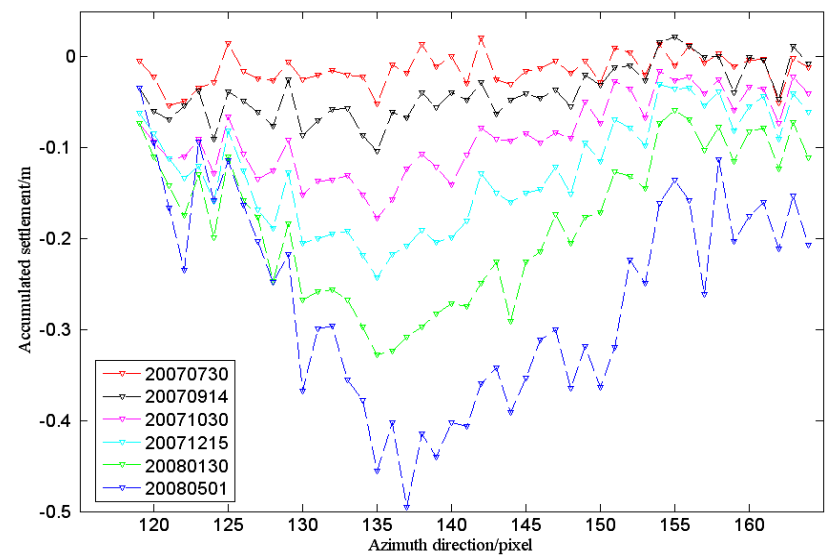

Figure5.Time series settlement profiles of subsidence funnel D in distance and azimuth direction

Also funnel $\mathrm{A}$ and $\mathrm{C}$ area has been analyzed respectively, as shown in figure 4 and figure 5 . The settlement of funnel $\mathrm{C}$ and funnel D area were continuous subsiding as times goes by, as funnel B area. But there was discontinuity of settlement in the 
space in the figure 6 and 7, due to the poor coherence caused by near to water and crops.

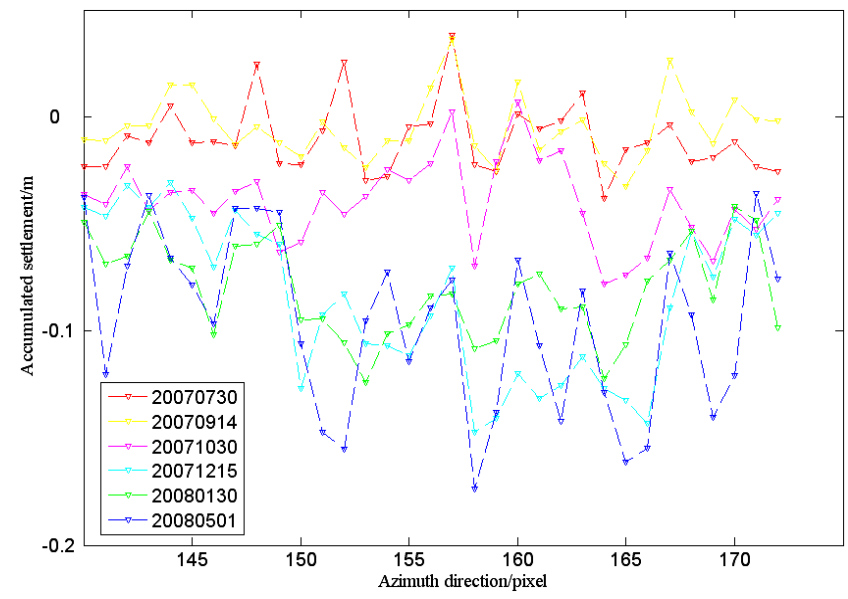

Figure 6.Time series settlement profile of subsidence funnel A in bearing direction

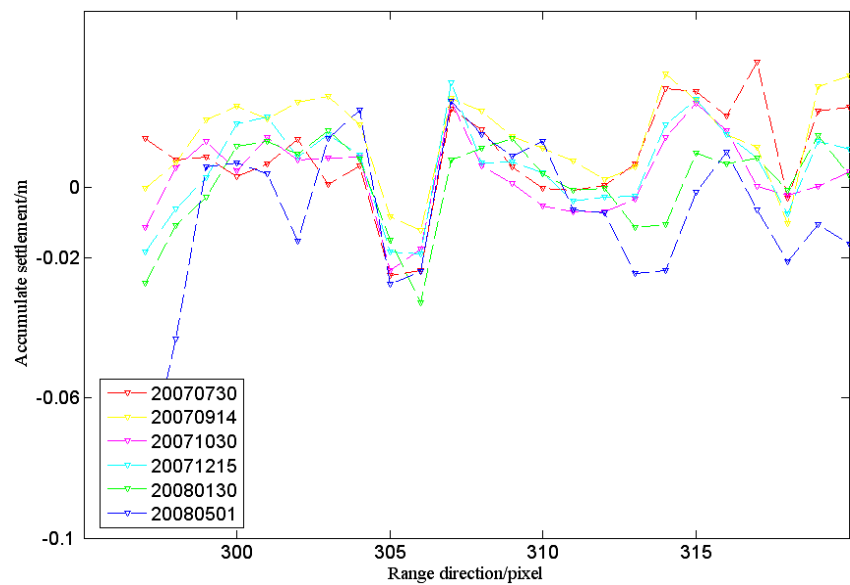

Figure 7.Time series settlement profile of subsidence funnel C in distance direction

\section{CONCLUTION}

In this paper SBAS method has been tested with 1-band PALSAR sensor radar data, and the experiment results showed that this method can effectively get the subsidence sequence diagram of coal mine area surface. Although SBAS technology has improved D-InSAR technology results well, the quantity of using data was limited, leading to a certain error in results. As the data have two scene data influenced by ice disaster, the overall coherence was poorer, and there were some deviation in unwrapping results, and the accuracy of inversion deformation has been affected. Due to the smaller mining area, and compared to the atmospheric error phase of space low frequency characteristics, settlement has higher spatial frequency, the differential interferometric phase was led by the phase signal of settlement, so in this paper the influence of atmospheric error hasn't been considered (Baowei Huang et al., 2012). In addition, the phase error caused by inaccuracy DEM has not been considered in the data processing, and it is the further step to do research on how will DEM error can be used as a parameter to estimate and separate effectively.

\section{ACKNOWLEDGMENTS}

This paper is supported by Natural Science Foundation of China (40874010), Natural Science Foundation of Jiangxi China (2012ZBAB216001).

\section{REFERENCES}

Y.F. Zhu, X.L. Ding, Z.W. Li, S.J. Zhou, 2012. Discussion on the Application of GPS Using in Marine Construction Survey, Journal of Computers. Finland, Vol.7, pp. 1663-1670.

Y.F. Zhu, X.L. Ding, Y. Luo, Z.W. Li, 2011. Analysis on the Application of GPS-RTK Technology using in Yangkou manmade Island in Nantong City, Journal of Networks. Finland, Vol.6, pp. 1444-1451.

H.J. Yin, J.J. Zhu, Z.W. Li, X.L. Ding, C.C. Wang, 2011. Ground Subsidence Monitoring in Mining Area Using DInSAR SBAS Algorithm, Acta Geodaeticaet Cartographica Sinica. China, Vol.40, pp.52-58.

Usai S., KLEES R., 1999. SAR Interfermetry on a Very long Time Scale:a study of the Interferometric Characteristics of Man_made Features, IEEE Transactions on Geoscience and Remote Sensing.U.S, Vol.37, pp.2118-2123.

Usai S., 2003. A Least Squares Database Aproach for SAR Interfermetry Data, IEEE Transactions on Geoscience and Remote Sensing. U.S, Vol.41, pp.753-760.

Ferretti A., Prati C., and Rocca F., 2000. Nonlinear Subsidence Rate Estimation Using Permanet Scatters in Differential SAR Interferometry, IEEE Transactions on Geoscience and Remote Sensing. U.S, Vol.38, pp.2202-2212.

Ferretti A., Prati C., and Rocca F., 2001. Permanent Scatters in SAR interferometry, IEEE Transactions on Geoscience and Remote Sensing. U.S, Vol.39, pp.8-20.

Berandino P., Fornaro G., Lanari R., 2002. A new algorithm for surface deformation monitoring based on small baseline differential interferograms. IEEE Transactions on Geoscience and Remote Sensing, 40(11):2375-2383.

Lanri R., Mora O., Manunta M., 2004. A small_baseline approach for investigating deformation on full_resolution differential SAR interferograms, IEEE Transations on Geoscience and Remote Sensing. U.S, Vol.42, pp.1377-1386.

H.Zhang, C.Wang, T.Wu, Y.X.Tang, 2009. Research on DInSAR method Based on the coherent target. Science publications, China .

H.L.Yang, J.H. Peng, D.X. Zhang, S.H. Li, 2012. Infuluence of orbital errosr on InSAR data processing, Journal of Geomatics Science and Technology. China, Vol.29, pp.118-121.

X.Y. Dong, 2011. Research on earthquake deformation monitoring Based on image matching technology, Central South University, China .

X.F.He, M.He, 2012. Earth observation data processing method and comprehensive measurement based on InSAR Technology. Science publications, China . 
B.W.Huang, X.G.Song, Z.J.Wang, X.J.San, 2012. Research on ground subsidence monitoring of Ge Ting coal mine based on
D-InSAR and GIS techniques, Geotechnical Investigation \& Surveying. China, Vol.4, pp.55-60. 